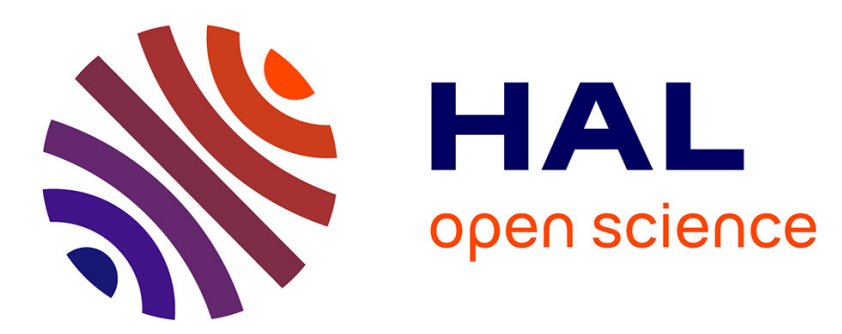

\title{
Effects of radiative transfer modelling on the dynamics of a propagating electrical discharge
}

Nicolas Kahhali, Philippe Rivière, Marie-Yvonne Perrin, Jean-Paul Gonnet, Anouar Soufiani

\section{- To cite this version:}

Nicolas Kahhali, Philippe Rivière, Marie-Yvonne Perrin, Jean-Paul Gonnet, Anouar Soufiani. Effects of radiative transfer modelling on the dynamics of a propagating electrical discharge. Journal of Physics D: Applied Physics, 2010, 43 (42), pp.425204. 10.1088/0022-3727/43/42/425204 . hal00569724

\section{HAL Id: hal-00569724 \\ https://hal.science/hal-00569724}

Submitted on 25 Feb 2011

HAL is a multi-disciplinary open access archive for the deposit and dissemination of scientific research documents, whether they are published or not. The documents may come from teaching and research institutions in France or abroad, or from public or private research centers.
L'archive ouverte pluridisciplinaire HAL, est destinée au dépôt et à la diffusion de documents scientifiques de niveau recherche, publiés ou non, émanant des établissements d'enseignement et de recherche français ou étrangers, des laboratoires publics ou privés. 


\title{
Effects of radiative transfer modelling on the dynamics of a propagating electrical discharge
}

\author{
Nicolas Kahhali, ${ }^{\dagger \S}$ Philippe Rivière ${ }^{\dagger}$ Marie-Yvonne Perrin, $^{\dagger}$ \\ Jean-Paul Gonnet, ${ }^{\ddagger}$ Anouar Soufiani ${ }^{\dagger}$ \\ † Laboratoire EM2C, CNRS UPR 288, École Centrale Paris, \\ 92295 Châtenay-Malabry Cedex, France \\ ${ }^{\ddagger}$ Schneider Electric, Power Business, LV Arc Breaking - Modeling \& Expertise \\ Site 38 EQI Eybens - 38050 GRENOBLE Cedex 9 \\ $\S$ Present address: Air Liquide - CRCD - Modeling group, 1 Chemin de la porte des \\ loges 78350 Les Loges En Josas \\ E-mail: Anouar.Soufiani@em2c.ecp.fr
}

\begin{abstract}
A radiative transfer methodology is developed for the modelling of coupled radiation, hydrodynamic and electromagnetic phenomena in unsteady air plasma flows. Absorption spectra are discretized according to the distribution functions of the absorption coefficients resulting from different types of radiative transitions, and this spectral model is combined with the differential $\mathrm{P}_{1}$ approximation which is shown to predict quite accurately radiative source terms. The study of a propagating electrical arc in a $2 \mathrm{D}$ channel shows that radiative transfer modelling affects significantly the shape of the plasma and its dynamics. In particular, when compared with the results from the net emission coefficient method, the arc velocity is found to increase due to radiation absorption in the arc boundaries.
\end{abstract}

\section{Introduction}

Radiative transfer in thermal plasmas, encountered for instance in electrical discharges, is an important mechanism in the local energy budget. It significantly 
contributes to the determination of the temperature field and, consequently, affects electrical and other transport properties of the medium. The accurate modelling of radiative fluxes and volumetric power remains a challenging task due to the spectral, spatial, directional and time dependence of radiative intensities.

Among the pioneering work in the field of plasma radiation modelling, Lowke [1] has calculated the temperature profile in a 1D low pressure sodium plasma by solving the exact radiative transfer equation (RTE) for the two Na lines and by coupling radiation, thermal conduction and Joule heating in the steady energy balance equation (ElenbaasHeller equation). Lowke and Capriotti [2] have carried out a similar study for high pressure air plasmas for which only continuum radiation was accounted for. They showed that, in these conditions, the $\mathrm{P}_{1}$ approximation was quite accurate in comparison with a ray-tracing method. Hermann and Schade [3] have used a line by line approach to solve the RTE equation coupled to the Elenbaas-Heller model for nitrogen steady plasmas. However, they considered atomic line and continuum radiation, but neglected molecular bound-bound transitions. In unsteady conditions, Lowke et al [4] have studied arc extinction for different gas mixtures (Ar, $\mathrm{N}_{2}, \mathrm{SF}_{6}, \mathrm{H}_{2}$, air) by either considering the medium as optically thin, or by neglecting atomic and molecular lines.

More recently, Gogel et al [5] used a Monte Carlo method to compute radiative transfer and then temperature fields in 1D axisymmetric air and ammonia plasmas. They neglected line contributions in the case of air plasmas and took into account some atomic lines for ammonia. Menart et al $[6,7]$ have combined a line by line approach with the discrete ordinates method to study $\mathrm{Ar}$ and $\mathrm{Ar}-\mathrm{Cu}$ plasma radiation in 2D cylindrical geometries. Due to the huge required computational times, they considered prescribed temperature fields and did not attempt to couple radiation to the energy balance equation. Such coupled calculations were undertaken by Deron et al [8] in 1D Ar plasmas at atmospheric pressure and in steady conditions. The results in terms of temperature profiles were favourably compared to spectroscopic measurements, and numerical simulations showed that the optically thin medium approximation leads to 
an underestimation of the maximum temperature of about $2000 \mathrm{~K}$.

The above listed studies show that the use of rigorous approaches for spectral radiative properties and RTE solvers remain limited to very simple geometries and generally to steady conditions. Several approximate methods have thus been developed to simplify the intricate calculations of radiative transfer. The partial characteristics method, initially introduced by Sevast'yanenko $[9,10]$, has been successfully applied in several studies as for instance in Refs. $[11,12,13]$ related to $\mathrm{SF}_{6}$ plasmas. This method remains however quite heavy due to the required beforehand tabulations and may introduce some inaccuracies resulting from the assumed linear temperature profile between each couple of exchanging volume elements.

For the geometrical part of radiative transfer calculations, the $\mathrm{P}_{1}$ approximation has been extensively used in association with different modelling levels of radiative properties. Reichert et al [14] and Rümpler et al [15] use band averaged absorption coefficients, with escape factors for resonance lines, to simulate arc propagation in complex geometries representative of electrical circuit breakers. The choice of a characteristic length for the escape factor remains however a crucial issue and may introduce important discrepancies [16]. Iordanidis et al [17] and Nordborg and Ionardis [18] have developed a k-distribution type model (see e.g. [19]) for $\mathrm{SF}_{6}$ plasmas. This spectral model enabled the simulation of propagating arcs in high voltage circuit breakers, including ablation phenomena [20] and complex geometries [21]. A similar approach has been developed by Lacombe et al [22] where self-absorbed atomic lines were gathered in a few lines for which the $\mathrm{P}_{1}$-RTE was solved spectrally to study an ICP torch flow.

Finally, the simplest method to include radiation in thermal plasma modelling is the net emission coefficient which can be simply tabulated versus temperature and directly used as a source term in the energy balance equation [23]. The accuracy of this very simple approach has been studied in Refs. [12, 24], and it is generally recognized that it can predict quite accurately radiative volumetric power and temperature level in the 
hottest parts of the plasma, but fails to predict radiation absorption in the boundaries and wall radiative fluxes. It remains however very popular and its simple implementation allows the treatment of unsteady plasmas in complex geometries (see e.g. Ref. [25]). A review of different applications in which the NEC is used is provided in Ref. [26]. However, to our knowledge, its accuracy when applied to unsteady propagating plasmas has never been studied.

The aim of the present work is to investigate the effects of radiative transfer modelling on the dynamics of a propagating electrical arc in air, initially at atmospheric pressure. We consider a 2D planar geometry and assume local thermodynamic and chemical equilibrium due to the high pressure level. Section 2 is devoted to radiation modelling. First, we develop a dedicated radiative property model based on the distribution function of the absorption coefficient and study the accuracy of the $\mathrm{P}_{1}$ approximation in the framework of this model. The hydrodynamic and electromagnetic model is described in Sec. 3 as well as the numerical tools used to solve the governing equations. Comparisons between the results from the NEC method and the more refined radiative transfer model are then presented and discussed in Sec. 4.

\section{Radiation models}

\subsection{Air plasma spectroscopic data}

An exhaustive spectroscopic database for air plasmas radiation was developed in previous studies $[27,28]$. Its accuracy was demonstrated in the temperature range 300-15000 $\mathrm{K}$ and in the wavenumber range $1000-150000 \mathrm{~cm}^{-1}$ by comparison with other theoretical databases and with available experimental results. The database included atomic bound-bound transitions of $\mathrm{N}, \mathrm{O}, \mathrm{N}^{+}, \mathrm{O}^{+}$and molecular bound-bound transitions concerning 19 electronic systems of $\mathrm{N}_{2}$ (first positive, second positive, BirgeHopfield 1, Birge-Hopfield 2, Carroll-Yoshino, Worley-Jenkins, Worley), $\mathrm{N}_{2}^{+}$(Meinel, first negative, second negative), $\mathrm{NO}\left(\gamma, \beta, \delta, \epsilon, \gamma^{\prime}, \beta^{\prime}, 11000 \AA\right.$, infrared $)$, and $\mathrm{O}_{2}$ (Schumann-Runge) systems. Details about the calculations of line intensities and 
positions for all rovibronic transitions belonging to these molecular systems are provided in Ref. [27]. Continuum contributions include $\mathrm{N}, \mathrm{O}, \mathrm{N}_{2}, \mathrm{O}_{2}$ and $\mathrm{NO}$ photoionization, $\mathrm{N}^{-}$ and $\mathrm{O}^{-}$photodetachment, $\mathrm{O}_{2}$ (Schumann-Runge) photodissociation and ion-electron, atom-electron and neutral molecule-electron Bremsstrahlung.

Atomic line positions and Einstein coefficients were taken from NIST database, which is complete enough for the temperature range considered here, and atomic photoionization cross sections were taken from TOPBASE database [29]. Details on atomic and continuum radiation data may be found in Ref. [28]. The broadening and the shift of atomic lines are calculated semi-classically as explained in Ref. [30]. Doppler and collisional effects are taken into account through a Voigt profile for all atomic and molecular lines.

The database developed in Refs. [27, 28] has been extended up to $25000 \mathrm{~K}$ by including bound-bound transitions of species $\mathrm{N}^{++}$and $\mathrm{O}^{++}$from the NIST compilation, the photoionisation of $\mathrm{N}^{+}$and $\mathrm{O}^{+}$from the TOPBASE data base [29], and the freefree transitions of electron $-\mathrm{N}^{++}$and electron - $\mathrm{O}^{++}$pairs; the spectral range has been extended up to $200000 \mathrm{~cm}^{-1}$. Absorption spectra have been then calculated in the temperature range $300-25000 \mathrm{~K}$ with a spectral increment varying linearly from $0.01 \mathrm{~cm}^{-1}$ at $1000 \mathrm{~cm}^{-1}$ to $0.157 \mathrm{~cm}^{-1}$ at $200000 \mathrm{~cm}^{-1}$ (about $310^{6}$ spectral points), which enables to correctly describe all absorption line shapes. This set of spectral absorption coefficients $\kappa_{\nu}(T)$ for various temperatures $T$ constitutes our reference model for radiative transfer calculations and for the calculation of approximate model parameters.

\subsection{Mixture absorption distribution function model}

Due to the huge required computational times, the high resolution approach using the above described set of absorption coefficients cannot be directly used in the coupled unsteady calculations considered in this study. An approximate spectral model is required. The Mixture Absorption Distribution Function (MADF) model was developed 
first in Ref. [31] for radiative transfer in air plasmas at temperatures above $10000 \mathrm{~K}$. It is extended here to temperatures down to $300 \mathrm{~K}$ in order to allow for diatomic molecular radiation which is important below $10000 \mathrm{~K}$. This model is based on the distribution function of the absorption coefficient $\kappa_{\nu}$, weighted by the Planck function

$$
\mathcal{F}\left(k, T_{P}, T\right)=\frac{\pi}{\sigma T_{P}^{4}} \int_{\nu / \kappa_{\nu}(T) \leq k} I_{\nu}^{0}\left(T_{P}\right) \mathrm{d} \nu
$$

which depends on two temperatures, $T_{P}$, used in the weighting Planck function $I_{\nu}^{0}$, and $T$ used to evaluate the plasma absorption coefficient. The use of this distribution function allows to replace spectral integrations by integrations over the possible values $k$ of the absorption coefficient. This approach is rigorous if the absorption coefficient $\kappa_{\nu}(T)$ satisfies the scaling approximation, i.e. if it can be written as the product of two independent functions $\kappa_{\nu}(T)=\eta(\nu) \times \Phi(T)$. However, the actual spectra do not satisfy this approximation and a splitting of different contributions, according to their dependence on $T$, is required. In practice, the absorption spectra have been split into three parts — called fictitious species - corresponding to (i) the contribution of molecules, (ii) the contribution of all the neutral atomic lines and the monoatomic continuum extending above $80,000 \mathrm{~cm}^{-1}$, (iii) the ionized atomic lines and the monoatomic continuum below $80,000 \mathrm{~cm}^{-1}$. This splitting ensures that quite all the points of the spectrum of a fictitious species have similar variations with temperature; therefore, considering the set of wave-numbers $\left\{\nu_{j}\left(k_{\text {ref }}^{\alpha}\right)\right\}$ at which the $\alpha^{\text {th }}$ fictitious absorption coefficient at a reference temperature $T_{\text {ref }}^{\alpha}$ takes the value $k_{\text {ref }}^{\alpha}$, we can assume that this absorption coefficient at another temperature $T$ will take a quite unique value for all the wave-numbers of this set; this enables to replace the sum on wave-numbers by a sum on the absorption coefficient values $k_{\text {ref }}^{\alpha}$ of each partial absorption spectrum $\alpha$ at the reference temperature $T_{\text {ref }}^{\alpha}$. The reference temperatures retained for the three fictitious species $\alpha=1,2$ and 3 are resp. 10,000 K, 13,000 K and 15,000 K. The absorption coefficient ranges retained are $10^{-3}-10^{3} \mathrm{~cm}^{-1}$ for species 1 and 3 and $10^{-2}-10^{3} \mathrm{~cm}^{-1}$ for species 2 . They have been chosen in order to accurately predict the emission due to the fictitious species for column lengths in the range $10^{-2}-10 \mathrm{~cm}$. These 
absorption coefficient ranges have been logarithmically discretized into five consecutive intervals $\left[k_{q}^{\alpha-}\left(T_{\text {ref }}^{\alpha}\right), k_{q}^{\alpha+}\left(T_{\text {ref }}^{\alpha}\right)\right], q=1 \ldots 5$. A sixth sub-range, indexed by $q=0$ and corresponding to the absorption coefficient values lower than $k_{1}^{\alpha-}\left(T_{\text {ref }}^{\alpha}\right)$ has been taken into account.

Since the scaling approximation for a given fictitious species is only approximate, the boundaries of the absorption coefficient ranges $\left[k_{q}{ }^{\alpha-}(T), k_{q}{ }^{\alpha+}(T)\right], q=0, \ldots, 5$, are calculated for a current temperature $T$ from the implicit correlation relation [32]

$$
\mathcal{F}_{\alpha}\left(k_{q}^{\alpha \pm}(T), T_{\text {ref }}^{\alpha}, T\right)=\mathcal{F}_{\alpha}\left(k_{q}^{\alpha \pm}\left(T_{\text {ref }}^{\alpha}\right), T_{\text {ref }}^{\alpha}, T_{\text {ref }}^{\alpha}\right)
$$

where $\mathcal{F}_{\alpha}$ is the distribution function defined following Eq. (1) for the $\alpha^{\text {th }}$ fictitious spectrum.

The first sub-ranges $\left[{k_{0}}^{\alpha-}(T),{k_{0}}^{\alpha+}(T)\right]$ have been assumed to be optically thin and thus are only characterized by the Planck mean values

$$
\widetilde{k_{0}^{\alpha}}(T)=\frac{\pi}{\sigma T^{4}} \int_{\nu / \kappa_{\nu}^{\alpha}(T) \leq k_{0}{ }^{\alpha+}(T)} \kappa_{\nu}^{\alpha}(T) I_{\nu}^{0}(T) \mathrm{d} \nu .
$$

Assuming that the discretization is fine enough so that absorption coefficients in each sub-range $q=1, . ., 5$ may be represented by a single value

$$
\widetilde{k_{q}^{\alpha}}(T)=\left[k_{q}^{\alpha-}(T)\right]^{\varpi_{\alpha}}\left[k_{q}^{\alpha+}(T)\right]^{1-\varpi_{\alpha}},
$$

where $\varpi_{\alpha}$ have to be optimized for each fictitious species, we obtain a model similar to the well-known Weighted Sum of Grey Gases model characterized by $6 \times 6 \times 6=216$ "grey gases" of absorption coefficients

$$
\widetilde{\kappa}_{q_{1} q_{2} q_{3}}(T)=\sum_{\alpha=1}^{3} \widetilde{k_{q_{\alpha}}^{\alpha}}(T)
$$

for all values of $q_{1}, q_{2}, q_{3}$ in the range $[0-5]$. The weights $a_{q_{1} q_{2} q_{3}}(T)$ to be associated to each of these 216 grey gases may be calculated from the joint distribution of the absorption coefficient according to

$$
a_{q_{1} q_{2} q_{3}}(T)=\frac{\pi}{\sigma T^{4}} \underset{\nu / k_{q_{\alpha}}^{\alpha-}(T) \leq \kappa_{\nu}^{\alpha}(T) \leq k_{q_{\alpha}}^{\alpha+}(T), \alpha=1 . .3}{\int} I_{\nu}^{0}(T) \mathrm{d} \nu,
$$


The use of this model consists in solving the radiative transfer equation for each triplet $\left(q_{1}, q_{2}, q_{3}\right)$ with absorption coefficients $\widetilde{\kappa}_{q_{1} q_{2} q_{3}}(T)$ and source terms $a_{q_{1} q_{2} q_{3}}(T) \times \sigma T^{4} / \pi$

$$
\frac{\partial I_{q_{1} q_{2} q_{3}}}{\partial s}=\widetilde{\kappa}_{q_{1} q_{2} q_{3}}\left(a_{q_{1} q_{2} q_{3}} \frac{\sigma T^{4}}{\pi}-I_{q_{1} q_{2} q_{3}}\right) .
$$

The total radiation intensity $I(s)$ is then simply given by adding the partial ones

$$
I(s)=\sum_{q_{1}, q_{2}, q_{3}=0}^{5} I_{q_{1} q_{2} q_{3}}(s) .
$$

It should be underlined that, for the boundary conditions associated to Eq. 7, the walls must be assumed gray since the set $\nu_{j}\left(\widetilde{\kappa}_{q_{1} q_{2} q_{3}}\right)$ associated to a given value of the absorption coefficient spreads over all the spectrum, and wall emissivity must remain constant for this set of wavenumbers.

Figure 2 shows comparisons between the total emissivities of uniform plasma columns, calculated from the high resolution line by line approach according to

$$
\epsilon_{t o t}^{L B L}(l, T)=\frac{\pi}{\sigma T^{4}} \int_{0}^{\infty}\left[1-\exp \left(-\kappa_{\nu}(T) l\right)\right] I_{\nu}^{0}(T) d \nu,
$$

and from the MADF model according to

$$
\epsilon_{\text {tot }}^{M A D F}(l, T)=\sum_{q_{1}, q_{2}, q_{3}=0}^{5} a_{q_{1} q_{2} q_{3}}(T)\left[1-\exp \left(-\widetilde{\kappa}_{q_{1} q_{2} q_{3}}(T) l\right)\right]
$$

as a function of $T$ for different column lengths $l$. The maximum observed differences between line by line and MADF emissivities remain limited to $5 \%$ in the considered temperature and optical path ranges.

[Figure 1 about here.]

\subsection{Net emission coefficient}

The net emission coefficient (NEC) is defined as

$$
\varepsilon_{N}\left(T, R_{p}\right)=\int_{0}^{\infty} \kappa_{\nu}(T) I_{\nu}^{0}(T) \exp \left(-\kappa_{\nu}(T) R_{p}\right) \mathrm{d} \nu
$$

The quantity $4 \pi \varepsilon_{N}\left(T, R_{p}\right)$ represents the divergence of the radiative flux vector at the center of an isothermal sphere of radius $R_{p}$ at temperature $T$. This quantity is also representative of the radiative power per unit volume escaping from a sphere of radius $R_{p}$. Figure 2 shows $\varepsilon_{N}$ for air plasmas at atmospheric pressure vs the temperature 
for various self-absorption lengths $R_{p}$, as calculated from the previously described spectroscopic database. This figure shows the strong self absorption of radiation since, for instance at $15000 \mathrm{~K}$, the emission is reduced by more than one order of magnitude for $R_{p}$ as small as $1 \mathrm{~mm}$. The net emission coefficient is simply used in the energy balance equation as a source term given by $P=-4 \pi \varepsilon_{N}\left(T, R_{p}\right)$.

[Figure 2 about here.]

\subsection{Accuracy of the $P_{1}$ approximation}

The $\mathrm{P}_{1}$ approximation transforms the directional radiative transfer equations into a second order elliptic partial differential equation where the unknown field is the zeroorder moment $G$ of the radiative intensity (see e.g. Ref. [33]). The simple form of the resulting equations makes it very attractive but the study of its validity under plasma conditions received little attention in the literature. We compare in this section the results from the $\mathrm{P}_{1}$ approximation and the exact ray tracing method for an air plasma confined in a 2D rectangular geometry with a prescribed temperature field. It is important to check its accuracy before using it in coupled calculations for the propagating electrical arc.

In the framework of the MADF model, the zero-order moment $G_{j}$, corresponding to the triplet $j \equiv\left(q_{1}, q_{2}, q_{3}\right)$, satisfies the equation

$$
\boldsymbol{\nabla} \cdot\left(-\frac{1}{3 k_{j}(T)} \nabla G_{j}\right)+k_{j}(T) G_{j}=k_{j}(T) G_{j}^{0}(T) .
$$

where $k_{j}=\widetilde{\kappa}_{q_{1} q_{2} q_{3}}$ and the source term $G_{j}^{0}$ is given by

$$
G_{j}^{0}(T)=4 a_{j}(T) \sigma T^{4}
$$

The associated Marshak boundary condition at an opaque diffuse and gray wall reads

$$
\frac{\epsilon_{w}}{2-\epsilon_{w}} G_{j}-\frac{2}{3 k_{j}(T)} \boldsymbol{n} \cdot \nabla G_{j}=\frac{\epsilon_{w}}{2-\epsilon_{w}} G_{j}^{0}\left(T_{w}\right),
$$

where $\epsilon_{w}$ is the wall emissivity and $\boldsymbol{n}$ is the normal to the wall directed inside the medium. The partial radiative flux $\boldsymbol{q}_{\boldsymbol{j}}$ and radiative source term $P_{j}$ in the energy 
equation are given by

$$
\begin{aligned}
\boldsymbol{q}_{j} & =-\frac{1}{3 k_{j}} \boldsymbol{\nabla} G_{j} \\
P_{j} & =-\boldsymbol{\nabla} \cdot \boldsymbol{q}_{\boldsymbol{j}}=-k_{j}(T)\left(G_{j}^{0}(T)-G_{j}\right) .
\end{aligned}
$$

The total associated quantities are simply given by summing over $j$.

In the ray tracing method used to check $\mathrm{P}_{1}, N_{v}$ rays are generated from each volume element and $N_{s}$ rays from each surface element, and the radiative transfer equation is integrated on each crossed cell for each ray. An iterative procedure is used in order to compute the wall leaving intensities from the incident intensities in the case of partially reflecting walls. In addition, integrations in the infinite direction (due to the 2D geometry considered here) are carried out semi-analytically using the functions [33]

$$
S_{i}(x)=\frac{2}{\pi} \int_{0}^{1} \frac{\mu^{3-i}}{\sqrt{1-\mu^{2}}} \exp \left(\frac{-x}{\mu}\right) d \mu .
$$

Converged results with ray tracing method were obtained for typically $N_{v}=100, N_{s}=50$ equally spaced directions for a $100 \times 100$ spatial grid mesh.

Calculations have been carried out for several rectangular geometries with a prescribed Gaussian temperature profile

$$
T(x, y)=2000+13000 \exp \left(-\alpha\left(x-x_{0}\right)^{2}-\beta\left(y-y_{0}\right)^{2}\right),
$$

with a maximum value at the point $\left(x_{0}, y_{0}\right)$ and with a decay characterized by the lengths $\alpha^{-1 / 2}$ and $\beta^{-1 / 2}$ in the $x$ and $y$ directions respectively. The cavity walls were assumed black at temperature $T_{w}=2000 \mathrm{~K}$.

Figure 3 shows examples of results for a square cavity of side length equal to $2 \mathrm{~cm}$ $\left(0 \leq x, y \leq 2 \mathrm{~cm}, x_{0}=y_{0}=1 \mathrm{~cm}\right)$ and for various confinement parameters $\alpha$ and $\beta$. The radiative volumetric power is quite accurately predicted with maximum errors of about $13 \%$ in the high temperature region. Although the integrated wall radiative flux is well predicted by the $\mathrm{P}_{1}$ approximation, its spatial distribution shows significant errors with important smoothing due to the diffusion-like approximation in the $\mathrm{P}_{1}$ method. Several tests with varying cavity aspect ratio, cavity size and plasma confinement showed 
similar results [34]. The radiative volumetric power is fairly well predicted by the $\mathrm{P}_{1}$ method while the wall flux distribution is strongly smoothed, especially for high cavity aspect ratios. As we are mostly interested in this study in the volumetric radiative power (we don't investigate for instance wall ablation due to radiation), we adopt the $\mathrm{P}_{1}$ approximation which reduces very significantly the CPU times required in coupled radiative/hydrodynamic/electromagnetic calculations. It is worth noting on Fig. 3 that the $\mathrm{P}_{1}$ approximation predicts quite accurately the radiative power in the absorbing regions of the plasma, while the use of the net emission coefficient cannot predict negative radiative powers.

[Figure 3 about here.]

\section{Hydrodynamic and electromagnetic model}

In the present section we briefly present the hydrodynamic and electromagnetic equations used to simulate the propagation of an electrical arc inside a channel between two planar electrodes. The 3D geometry shown in Fig. 4 is reduced to a $2 \mathrm{D}$ geometry (plane $(x, y))$ by assuming invariance of all the variables along the vertical axis $z$.

\subsection{Hydrodynamic equations}

The medium (initially air at room temperature and atmospheric pressure) is assumed to remain at macroscopic electro-neutrality and to be at local thermodynamic and chemical equilibrium. The local temperature determines thus the local chemical composition and transport properties of the plasma.

[Figure 4 about here.]

The conservation laws of mass, momentum and enthalpy write under these assumptions

$$
\begin{aligned}
& \frac{\partial \rho}{\partial t}+\nabla \cdot(\rho \boldsymbol{v})=0 \\
& \rho\left(\frac{\partial}{\partial t}+\boldsymbol{v} \cdot \boldsymbol{\nabla}\right) \boldsymbol{v}=-\boldsymbol{\nabla} p+\boldsymbol{j} \wedge \boldsymbol{B}+\boldsymbol{\nabla} \cdot \overline{\bar{\Pi}}
\end{aligned}
$$




$$
\rho\left(\frac{\partial}{\partial t}+\boldsymbol{v} \cdot \boldsymbol{\nabla}\right) h=\boldsymbol{\nabla} \cdot(\lambda \nabla T)+\left(\frac{\partial}{\partial t}+\boldsymbol{v} \cdot \boldsymbol{\nabla}\right) p+\boldsymbol{j} \cdot \boldsymbol{E}+P
$$

where conventional notations are used for the velocity $\boldsymbol{v}$, the density $\rho$, the pressure $p$, the electrical current density $\boldsymbol{j}$, the electric $(\boldsymbol{E})$ and magnetic $(\boldsymbol{B})$ fields, and the enthalpy per unit mass $h . P$ in the enthalpy equation designates the radiative source term calculated either from the net emission coefficient or from the $\mathrm{P}_{1}-\mathrm{MADF}$ model. The elements of the stress tensor $\overline{\bar{\Pi}}$ in Eq. 20 are given by

$$
\Pi_{i k}=\mu\left(\frac{\partial v_{i}}{\partial x_{k}}+\frac{\partial v_{k}}{\partial x_{i}}-\frac{2}{3} \delta_{i k} \frac{\partial v_{j}}{\partial x_{j}}\right)
$$

where $\mu$ is the plasma viscosity.

\subsection{Electromagnetic model}

In order to simplify Maxwell's equations which govern the electromagnetic fields, we use the commonly admitted approximations for electrical continuous discharges, namely a quasi-stationary regime and a small magnetic Reynolds number (see e.g. [35]). The electric field can then be obtained from a scalar potential $V$ and the Ohm law writes simply

$$
\boldsymbol{j}=-\sigma \nabla V=\sigma \boldsymbol{E}
$$

The magnetic field at point $M$, generated by the electrical current, is calculated from the Biot-Savart law

$$
\boldsymbol{B}(M)=\frac{\mu_{0}}{4 \pi} \iiint_{S \in \Omega} \frac{\boldsymbol{j}(S) \wedge \boldsymbol{S} \boldsymbol{M}}{\|\boldsymbol{S} \boldsymbol{M}\|^{3}} \mathrm{~d} \Omega
$$

where $\mu_{0}$ is the magnetic permeability and the integration is carried out over all the studied volume. In practice, a total sinusoidal electrical current $i(t)$ is prescribed

$$
i(t)=I_{0} \sin (2 \pi f t+\phi)
$$

and the spatially uniform electric field is deduced from

$$
\boldsymbol{E}(t)=-\frac{i(t)}{\iint_{\mathcal{P}} \sigma(x, y) \mathrm{d} x \mathrm{~d} y} \boldsymbol{e}_{z} .
$$


The magnetic field is calculated as the sum of a contribution $B_{e}$ from the electrodes and a contribution from the electrical arc itself $B_{a}$. If we consider a plane $(x, y)$ at mid-height between the electrodes, $B_{e}$ is given by

$$
\boldsymbol{B}_{e}(x, y, t)=\left(\frac{\mu_{0} i(t)}{4 \pi(h / 2-z)}+\frac{\mu_{0} i(t)}{4 \pi(h / 2+z)}\right) \boldsymbol{e}_{y}=\frac{\mu_{0} i(t)}{\pi h} \boldsymbol{e}_{y},
$$

where $h$ is the distance between the electrodes. The contribution $B_{a}$ of the plasma itself is calculated from Equ. 24 where the integral is restricted to plasma volume. As the present calculations are two-dimensional, the 3D integral reduces to a simpler $2 \mathrm{D}$ integral in the calculation plane.

\subsection{Numerical methods}

The hydrodynamic equations for the compressible fluid were solved using the software NS2 previously developed and validated for the 2D simulation of electrical arcs, and in particular those involving circuit breakers [36, 37]. A time splitting method is used with a finite element discretization for the diffusion step and a finite volume formulation for the convective step. In this second step, a Van Leer flux splitting method (see e.g. Ref. [38]) is adopted in order to accurately capture discontinuities due to possible shock waves or to plasma front waves. A quadrilateral mesh with 40000 cells is used as shown in Fig. 5 .

[Figure 5 about here.]

Concerning radiative transfer calculations with the $\mathrm{P}_{1}$-MADF model, a finite element method was developed to solve Eqs. 13 and 14 on the same mesh, and was coupled to the hydrodynamic solver. As these equations involve both absorption coefficients $k_{j}$ and their inverse values, and, due to the very wide range of variation of the $k_{j}$ 's, the matrices obtained from the finite element method are badly conditioned. A Conjugate gradient method with Jacobi preconditioning was used to solve the resulting algebraic systems of equations. The coupling between radiation and hydrodynamic calculations is done explicitly. It only involves the exchange of temperature profiles and radiative 
source terms between the two modules with a given frequency (multiple of the time step used for the integration of hydrodynamic equations). Varying the frequency between 1 and 50 did not show significant differences since very small time steps (of about $10^{-8} \mathrm{~s}$ ) were required for the hydrodynamic equations.

The transport properties, namely, the temperature and pressure dependent electrical conductivity, thermal conductivity and viscosity, of air plasmas are taken from Ref. [39].

\section{Propagation of an electrical discharge}

The considered geometry is that of a rectangular channel of width $1.4 \mathrm{~cm}$ and length $9.4 \mathrm{~cm}$, connected to an exhaust area through a small nozzle (Figure 5). The distance $h$ between the electrodes is assumed to be equal to $2.4 \mathrm{~cm}$. The initial temperature and pressure in the medium (air at rest) are $300 \mathrm{~K}$ and 1 atm respectively. The plasma ignition is numerically simulated by prescribing an energy source term with a Gaussian shape centered at $(x=2.1, y=0.7 \mathrm{~cm})$, a maximum value of $2.10^{11} \mathrm{~W} \cdot \mathrm{m}^{-3}$, and a standard deviation of the Gaussian equal to $0.3 \mathrm{~cm}$. During this non-physical ignition stage, the momentum equation is solved but the temperature field is calculated without radiation and Joule heating, and its maximum value rises up to $15000 \mathrm{~K}$ between times $t=0$ and $t=43.9 \mu \mathrm{s}$. At this last time, the electrical conductivity in the heated region is sufficiently high to maintain the electrical discharge. The source term is then dropped and the coupled simulations begin with the two different radiation models $\left(\mathrm{P}_{1^{-}}\right.$ MADF and NEC) and with a total current intensity characterized by $I_{0}=12000 \mathrm{~A}$, $\phi=0.094 \mathrm{rad}$, and $f=50 \mathrm{~Hz}$ in Eq. 25.

Calculation with the net emission coefficient are carried out with the value $R_{p}=$ $1 \mathrm{~mm}$ which provided values of the maximum temperature level similar to those from the $\mathrm{P}_{1}$-MADF model, although this value is smaller than the calculated characteristic length of the arc.

Figures 6 to 10 show comparisons between the temperature, pressure, density and 
current density profiles obtained respectively at $t=50,140,210,360$ and $750 \mu$ s along the channel central axis $(y=0.7 \mathrm{~cm})$ with $\mathrm{NEC}$ and $\mathrm{P}_{1}$-MADF models. Figures 11 and 12 show the 2D temperature and radiative source term fields obtained at the same times.

During the ignition stage, plasma expansion is observed with high pressures and low densities in the heated zone. Two pressure waves are created and the one traveling towards the dead end of the channel reaches the wall approximately at $t=50 \mu$ s (Fig. 6). At that moment, the velocity of the pressure wave traveling towards the nozzle is about $350 \mathrm{~m} \cdot \mathrm{s}^{-1}$. Figures 6 and 11 show that the temperature fields calculated with NEC and $\mathrm{P}_{1}$-MADF models at $t=50 \mu \mathrm{s}$ are very similar since radiation calculations only began $6.1 \mu \mathrm{s}$ prior to that moment.

At $t=140 \mu \mathrm{s}$, the plasma has moved towards the exit nozzle under Laplace forces and the pressure wave reaches the channel exit where it is reflected. The high temperature region is more spread when calculated with the $\mathrm{P}_{1}$-MADF model (Figs. 7 and 11) and the arc is slightly in advance. Also, the pressure level is significantly higher when computed with the NEC model. Figure 12 shows that the $\mathrm{P}_{1}$-MADF model predicts a strong radiation absorption in the region surrounding the arc, which cannot be predicted by the NEC method. The absorption level reaches $2.510^{10} \mathrm{~W} . \mathrm{m}^{-3}$, which is of the same order as the maximum emission level.

Both reflected pressure waves reach the arc approximately at $t=210 \mu \mathrm{s}$. The result is to shear it and to split it into two parts as shown in Fig. 11. The arc mean velocity between $t=50$ and $t=210 \mu \mathrm{s}$ is about $220 \mathrm{~m} \cdot \mathrm{s}^{-1}$ when calculated with the $\mathrm{P}_{1}$-MADF model and about 180 m.s ${ }^{-1}$ with the NEC method. Radiation absorption in front of the discharge increases the temperature level and enhances the electrical conductivity, which in return increases arc velocity. This mechanism, which is also important for streamer propagation (see e.g. Ref.[40]), cannot be accounted for with the NEC method. In the same manner, radiative transfer with absorption leads naturally to a wider high temperature region as observed in Figs. 11. Current density profiles 
follow the temperature profiles with a wider shape when predicted with the $\mathrm{P}_{1}$-MADF model, but as the total current is prescribed, the maximum local current intensities are obtained with the NEC method. Due to constriction forces, the arc recombines after a complex stage of pressure wave reflections and is then pushed towards the channel exit which is reached approximately at $t=870 \mu \mathrm{s}$ with the $\mathrm{P}_{1}$-MADF model, and $t=1070 \mu \mathrm{s}$ with the NEC method. These times correspond to a maximum temperature equal to $10000 \mathrm{~K}$ at the entrance of the nozzle.

The results presented above were obtained with a time step for radiation calculations equal to the time step for flow calculation. Table 1 shows a comparison between the required CPU times for the full calculations $(0<t<1200 \mu \mathrm{s})$ with $\mathrm{P}_{1^{-}}$ MADF and NEC models. Also are shown the CPU times for a frequency of radiation calculations equal to 5 or 50 , and with an optimized MADF model (36 ranges of absorption coefficient instead of 216 [34]), which didn't modify significantly the results. Table 1 shows that reasonably accurate radiative transfer calculations may be carried out with CPU times only two times greater than those required by the flow field calculation.

[Table 1 about here.]

[Figure 6 about here.]

[Figure 7 about here.]

[Figure 8 about here.]

[Figure 9 about here.]

[Figure 10 about here.]

[Figure 11 about here.]

[Figure 12 about here.] 


\section{Conclusion}

Facing the complexity of radiative transfer calculations in unsteady plasma flows, we have developed a method that seems optimal in terms of compromise between accuracy and computational capabilities. The method is based on the representation of the complex plasma absorption and emission spectra by the distribution function of the absorption coefficient which presents smoother variations than the absorption coefficient itself. The MADF model enables accurate radiation calculations by solving 216 equations instead of about three million equations required in a full high resolution approach. MADF model parameters were constructed from previously established fundamental spectroscopic data for the various radiation mechanisms involved in air plasmas. For the geometrical part of radiative transfer predictions, the analysis of the $\mathrm{P}_{1}$ approximation in the framework of plasma radiation shows that the volumetric source term is quite accurately predicted, while wall fluxes are significantly smoothed.

The combination of MADF and $\mathrm{P}_{1}$ approximations allowed to study the dynamics of a propagating electrical discharge, and to compare the results with those from the popular net emission coefficient method. The same hydrodynamic and electromagnetic model was coupled to radiative transfer calculations with both radiation models. It is found that, while the arc maximum temperature level may be roughly predicted by the NEC method through the choice of a convenient characteristic self-absorption length $R_{p}$, the temperature distribution, hydrodynamic and electrical characteristics of the propagating arc are very sensitive to radiative transfer modelling. In particular, higher arc velocities are obtained when the radiation model can capture a correct positive source term in the absorbing regions and the spread of the hot regions is also larger. These effects on arc dynamics are important for electrical circuit breaker modelling, among other applications. Comparisons between computational times with different models show that quite accurate radiative transfer calculations can be used at a reasonable cost.

Acknowledgments: The authors acknowledge J.-Y. Battandier for his valuable help 
in the implementation of $\mathrm{P}_{1}$ equations into the NS2 software. 
[1] J.J. Lowke. A relaxation method of calculating arc temperature profiles applied to discharges in sodium vapor. Journal of Quantitative Spectroscopy and Radiative Transfer, 9:839-854, 1969.

[2] J.J. Lowke and E.R. Capriotti. Calculation of temperature profiles of high pressure electric arcs using the diffusion approximation for radiation transfer. Journal of Quantitative Spectroscopy and Radiative Transfer, 9:207-236, 1969.

[3] W. Hermann and E. Schade. Radiative energy balance in cylindrical nitrogen arcs. Journal of Quantitative Spectroscopy and Radiative Transfer, 12:1257-1282, 1972.

[4] J.J. Lowke, R.E. Voshall, and H.C. Ludwig. Decay of electrical conductance and temperature of arc plasmas. Journal of Physics D: Applied Physics, 44:3513-3523, 1973.

[5] T.H. Gogel, A. Sedghinasab, and D.R. Kiefer. Radiation transfer computation in cylindrical arc columns using a monte carlo method. Journal of Quantitative Spectroscopy and Radiative Transfer, 52:179-194, 1994.

[6] J. Menart, J. Heberlein, and E. Pfender. Line-by-line method of calculating emission coefficients for thermal plasmas consisting of monoatomic species. Journal of Quantitative Spectroscopy and Radiative Transfer, 56:377-398, 1996.

[7] J. Menart. Radiative transport in a two-dimensional axisymmetric thermal plasma using the $s-n$ discrete ordinate method on a line by line basis. Journal of Quantitative Spectroscopy and Radiative Transfer, 67:273-291, 2000.

[8] C. Deron, Ph. Rivière, M-Y. Perrin, and A. Soufiani. Coupled radiation, conduction and joule heating in argon thermal plasma. Journal of Thermophysics and Heat Transfer, 20(2):211-219, 2006.

[9] V. Sevast'yanenko. Radiation transfer in a real spectrum. integration over frequency. $J$. Engineering Physics, 36:138-148, 1979.

[10] V. Sevast'yanenko. Radiation transfer in a real spectrum. integration with respect to the frequency and angles. J. Engineering Physics, 38:173-179, 1980.

[11] V. Aubrecht and J.J. Lowke. Calculations of radiation transfer in $\mathrm{SF}_{6}$ plasmas using the method of partial characteristics. Journal of Physics D: Applied Physics, 27:2066-2073, 1994.

[12] G. Raynal and A. Gleizes. Radiative transfer calculation in $\mathrm{SF}_{6}$ arc plasmas using partial characteristics. Plasma Sources Sci. Technol., 4(1):152-160, 1995.

[13] G. Raynal, P.J. Vergne, and A. Gleizes. Radiative transfer in $\mathrm{SF}_{6}$ and $\mathrm{SF}_{6}-\mathrm{Cu}$ arcs. Journal of Physics D: Applied Physics, 28:508-515, 1995.

[14] F. Reichert, Ch. Rümpler, F. Berger, H. Stammberger, and P. Terhoeven. Simulation of the radiative transfer in air plasma flows. In Proceedings of the 17th Symposium on Physics of Switching Arc,Nove Mesto na Morave, Czech Republic, pages 177-180, 2007. 
[15] Ch. Rümpler, F. Reichert, F. Berger, H. Stammberger, and P. Terhoeven. Simulation and verification of the arc behaviour in quenching chambers with splitter plates. In Proceedings of the 17th Symposium on Physics of Switching Arc,Nove Mesto na Morave, Czech Republic, pages 181-184, 2007.

[16] Ch. Deron, Ph. Rivière, M.-Y. Perrin, and A. Soufiani. Modelling radiative properties of LTE air atmospheric plasmas. In Proceedings of the 15th International Conference on Gas Discharges and their Applications, Toulouse, pages 145-148, 2004.

[17] A. Iordanidis, H. Nordborg, and Ch. Franck. Radiation based $\mathrm{SF}_{6}$ and PTFE arc simulations. In Proceedings of the 17th Symposium on Physics of Switching Arc,Nove Mesto na Morave, Czech Republic, pages 121-124, 2007.

[18] H. Nordborg and A.A. Iordanidis. Self-consistent radiation based modelling of electric arcs: I. Efficient radiation approximations. Journal of Physics D-Applied Physics, 41(13):135205, 2008.

[19] H.R. Goody and Y. Yung. Atmospheric Radiation. Oxford University Press, 1989.

[20] A.A. Iordanidis and C.M. Franck. Self-consistent radiation-based simulation of electric arcs: II. Application to gas circuit breakers. Journal of Physics D-Applied Physics, 41(13):135206, 2008.

[21] A.A. Iordanidis and C.M. Franck. Self-consistent simulation of arcs in realistic non-cylindrical breaker nozzles. In Proceedings of the 17th International Conference on Gas Discharges and their Applications, Cardiff, 2008.

[22] J.G. Lacombe, Y. Delannoy, and C. Trassy. The role of radiation in modelling of argon inductively coupled plasmas at atmospheric pressure. Journal of Physics D: Applied Physics, 41(16):165204 (9pp), 2008.

[23] J.J. Lowke. Predictions of arc temperature profiles using approximate emission coefficients for radiation losses. Journal of Quantitative Spectroscopy and Radiative Transfer, 14:111-122, 1974.

[24] S.D. Eby, J.Y. Trépanier, and X.D. Zhang. Modelling radiative transfer in $\mathrm{SF}_{6}$ circuit-breaker arcs with the P-1 approximation. Journal of Physics D: Applied Physics, 31(13):1578-1588, 1998.

[25] B. Swierczynski, J. J. Gonzalez, P. Teulet, P. Freton, and A. Gleizes. Advances in low-voltage circuit breaker modelling. Journal of Physics D: Applied Physics, 37(4):595-609, 2004.

[26] A. Gleizes, J.J. Gonzalez, and P. Freton. Thermal plasma modelling - topical review. Journal of Physics D: Applied Physics, 38:R153-R183, 2005.

[27] S. Chauveau, M.-Y. Perrin, Ph. Rivière, and A. Soufiani. Contributions of diatomic molecular electronic systems to heated air radiation. JQSRT, 72:503-530, 2002.

[28] S. Chauveau, Ch. Deron, M.-Y. Perrin, Ph. Rivière, and A. Soufiani. Radiative transfer in LTE air plasmas for temperatures up to $15000 \mathrm{~K}$. JQSRT, 77:113-130, 2003.

[29] The Opacity Project Team. The Opacity Project, volume 1. Institute of Physics Publishing, 
Bristol and Philadelphia, 1995.

[30] Ph. Rivière. Systematic semi-classical calculations of stark broadening parameters of NI, OI, NII, OII multiplets for modelling the radiative transfer in atmospheric air mixture plasmas. Journal of Quantitative Spectroscopy and Radiative Transfer, 73:91-110, 2002.

[31] Ph. Rivière, A. Soufiani, M-Y. Perrin, H. Riad, and A. Gleizes. Air mixture radiative property modelling in the temperature range $10000 k-40000 k$. Journal of Quantitative Spectroscopy and Radiative Transfer, 56:29-45, 1996.

[32] M.K. Denison and B.W. Webb. The spectral-line-based Weigthed-Sum-of-Gray-Gases model in nonisothermal nonhomogeneous media. J. Heat Transfer, 117:359-365, 1995.

[33] M. F. Modest. Radiative heat transfer. Academic Press, 2003.

[34] N. Kahhali. Modélisation et simulations numériques du transfer radiatif dans des plasmas d'arc électrique. Thèse de doctorat, École Centrale Paris, 2009.

[35] J.-L. Delcroix and A. Bers. Physique des plasmas, vol. 2. CNRS Editions, Paris, 1994.

[36] P. Chévrier, M. Barrault, and C. Fiévet. Hydrodynamic model for electrical arc modelling. IEEE Transactions on Power Delivery, 11:1824-1828, 1996.

[37] E. Doméjean, P. Chévrier, C. Fiévet, and P. Petit. Arcwall interaction modelling in a low-voltage circuit breaker. J. Phys. D: Appl. Phys., 30:2132-2142, 1997.

[38] C. Hirsch. Numerical computation of internal and external flows, Volume 2. Elsevier, 1999.

[39] J. Bacri and S. Raffanel. Calculation of transport coefficients of air plasmas. Plasma Chemistry and Plasma Processing, 9(1):133-154, 1989.

[40] S.V. Pancheshnyi, S.M. Starikovskaia, and A.Y. Starikovskii. Role of photoionization processes in propagation of cathode-directed streamer. Journal of Physics D: Applied Physics, 34:1-11, 2001. 


\section{List of Figures}

1 Total emissivities of uniform air plasma columns at atmospheric pressure. Column lengths are 0.01, 0.05, 0.1, 0.2, 0.5, 1, 2, 5 and $10 \mathrm{~cm}$. . . . . 23

2 Net emission coefficient of air plasmas at atmospheric pressure and different values of $R_{p} \ldots \ldots \ldots \ldots$. . . . . . . . . . . . 24

3 Divergence of the radiative flux vector (upper part) along the central line $x=1 \mathrm{~cm}$ inside a square cavity of $2 \mathrm{~cm}$ side length with (a) $\alpha=\beta=2 \mathrm{~cm}^{-2}$, (b) $\alpha=\beta=3 \mathrm{~cm}^{-2}$, (c) $\alpha=\beta=5 \mathrm{~cm}^{-2}$, and wall radiative flux (lower part). $\mathrm{P}_{1}$ results are in dashed lines. . . . . . . . . . . . . . . . 25

4 The $(x, y)$ plane used for $2 \mathrm{D}$ transient simulations. . . . . . . . . . . 26

5 The geometry and grid mesh used for the coupled 2D simulations . . . . 27

6 Temperature, pressure, density and current density profiles along the central channel axis calculated with the two radiation models at $t=50 \mu s 28$

7 Temperature, pressure, density and current density profiles along the central channel axis calculated with the two radiation models at $t=140 \mu s 29$

8 Temperature, pressure, density and current density profiles along the central channel axis calculated with the two radiation models at $t=210 \mu \mathrm{s} 30$

9 Temperature, pressure, density and current density profiles along the central channel axis calculated with the two radiation models at $t=360 \mu s 31$

10 Temperature, pressure, density and current density profiles along the central channel axis calculated with the two radiation models at $t=750 \mu \mathrm{s} 32$

11 Temperature field computed with NEC (left) and $\mathrm{P}_{1}-\mathrm{MADF}$ (right) models at different times. . . . . . . . . . . . . . . . . . 33

12 Radiative volumetric power computed with $\mathrm{NEC}$ (left) and $\mathrm{P}_{1}$-MADF (right) models at different times. . . . . . . . . . . . . . . . 34 


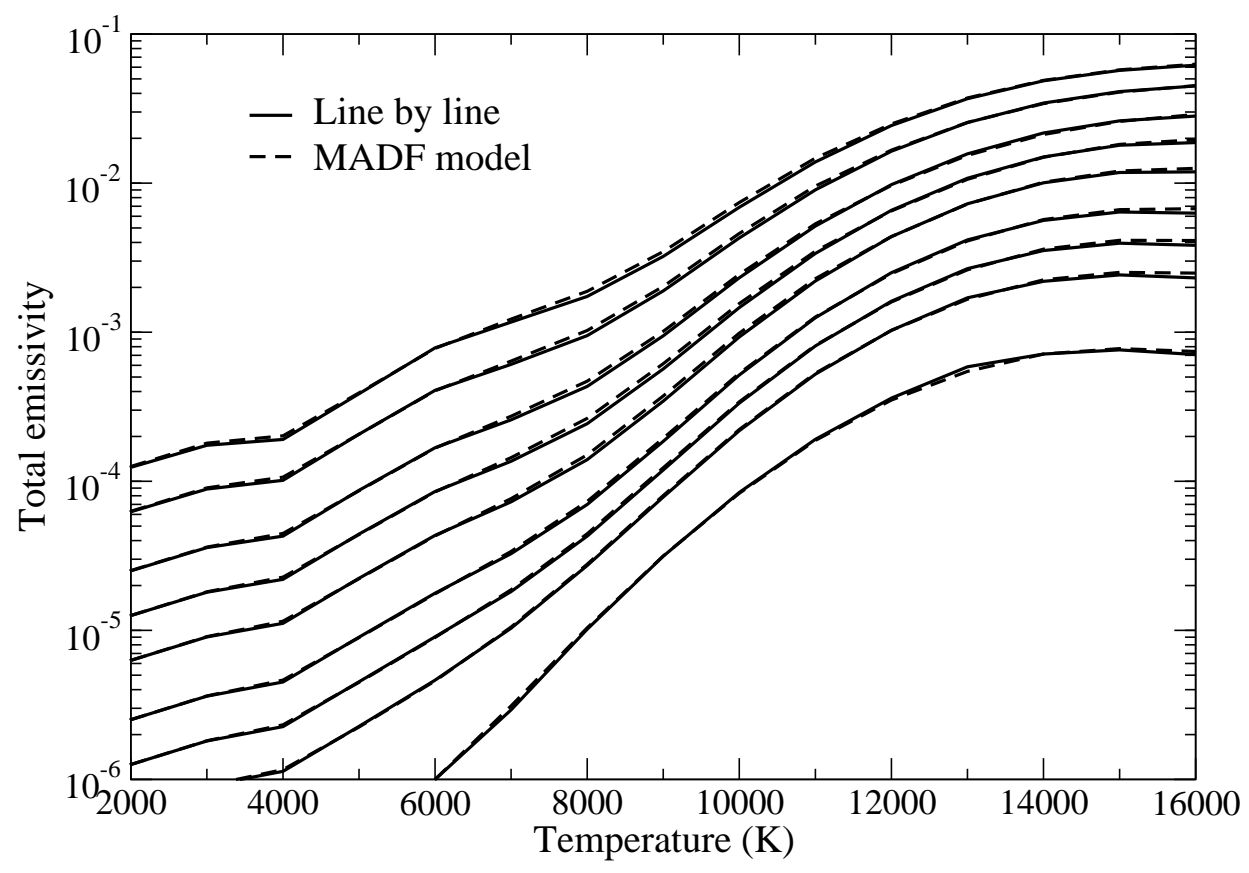

Figure 1: Total emissivities of uniform air plasma columns at atmospheric pressure. Column lengths are 0.01, 0.05, 0.1, 0.2, 0.5, 1, 2, 5 and $10 \mathrm{~cm}$. 


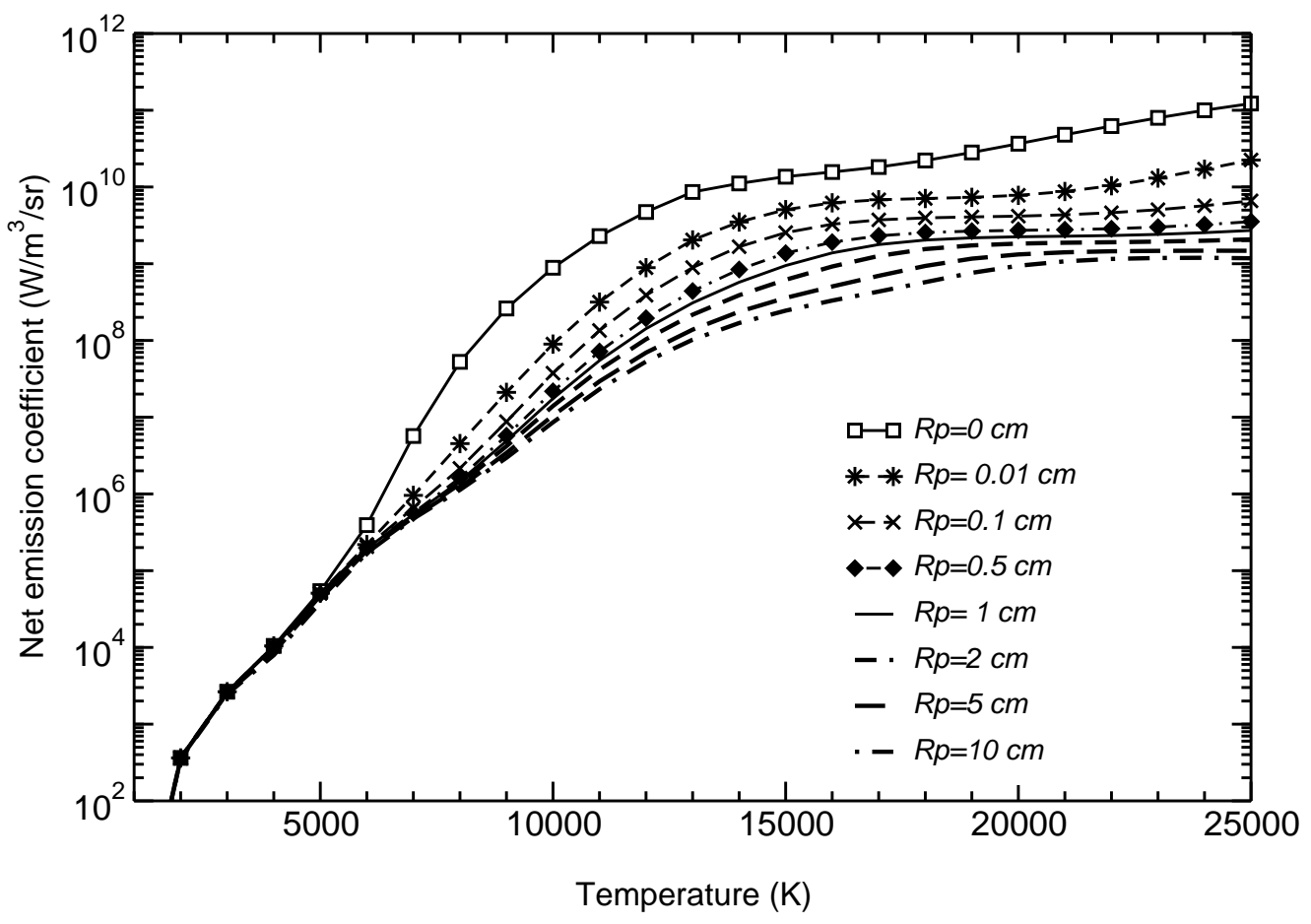

Figure 2: Net emission coefficient of air plasmas at atmospheric pressure and different values of $R_{p}$. 

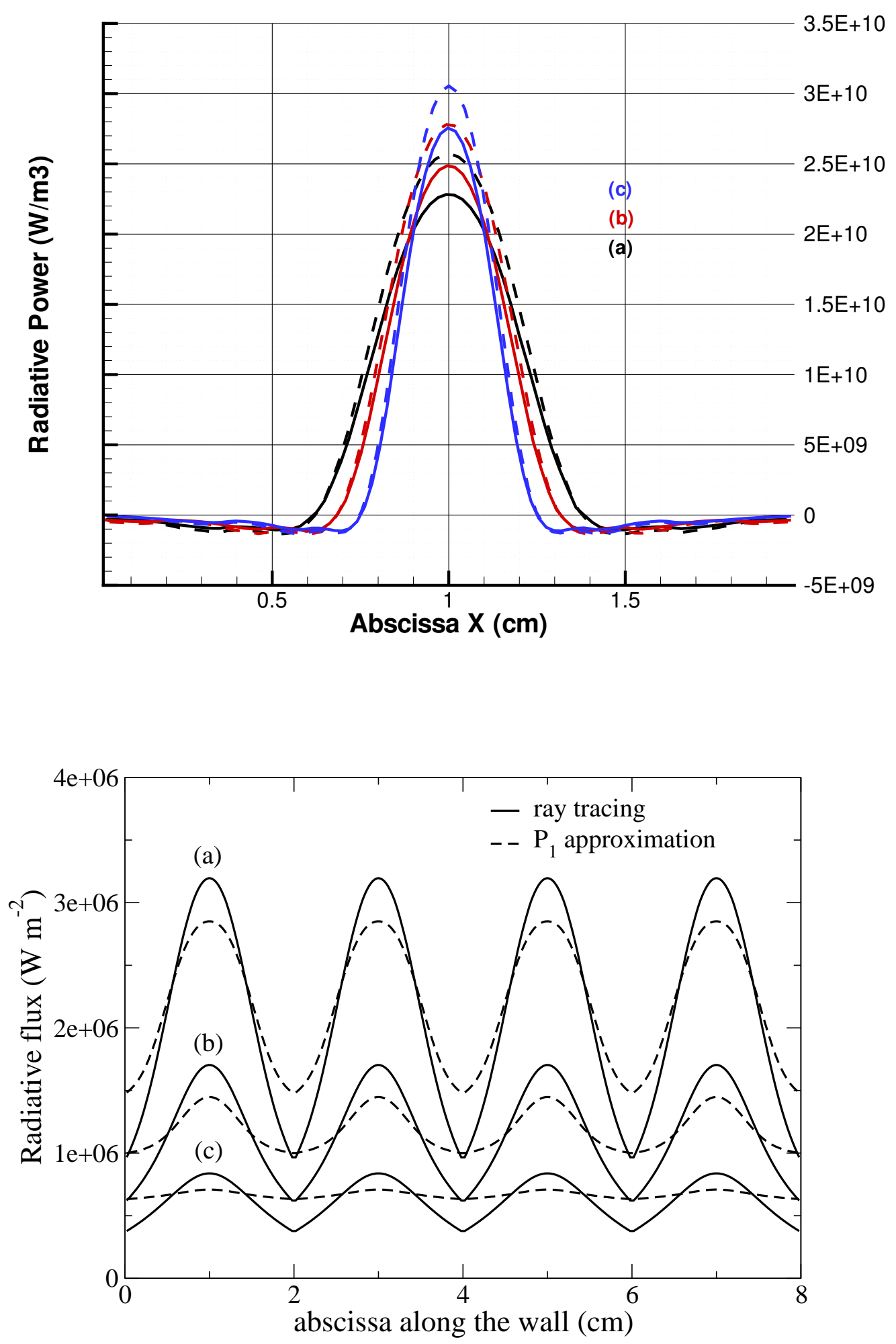

Figure 3: Divergence of the radiative flux vector (upper part) along the central line $x=1 \mathrm{~cm}$ inside a square cavity of $2 \mathrm{~cm}$ side length with (a) $\alpha=\beta=2 \mathrm{~cm}^{-2}$, (b) $\alpha=\beta=3 \mathrm{~cm}^{-2}$, (c) $\alpha=\beta=5 \mathrm{~cm}^{-2}$, and wall radiative flux (lower part). $\mathrm{P}_{1}$ results are in dashed lines. 


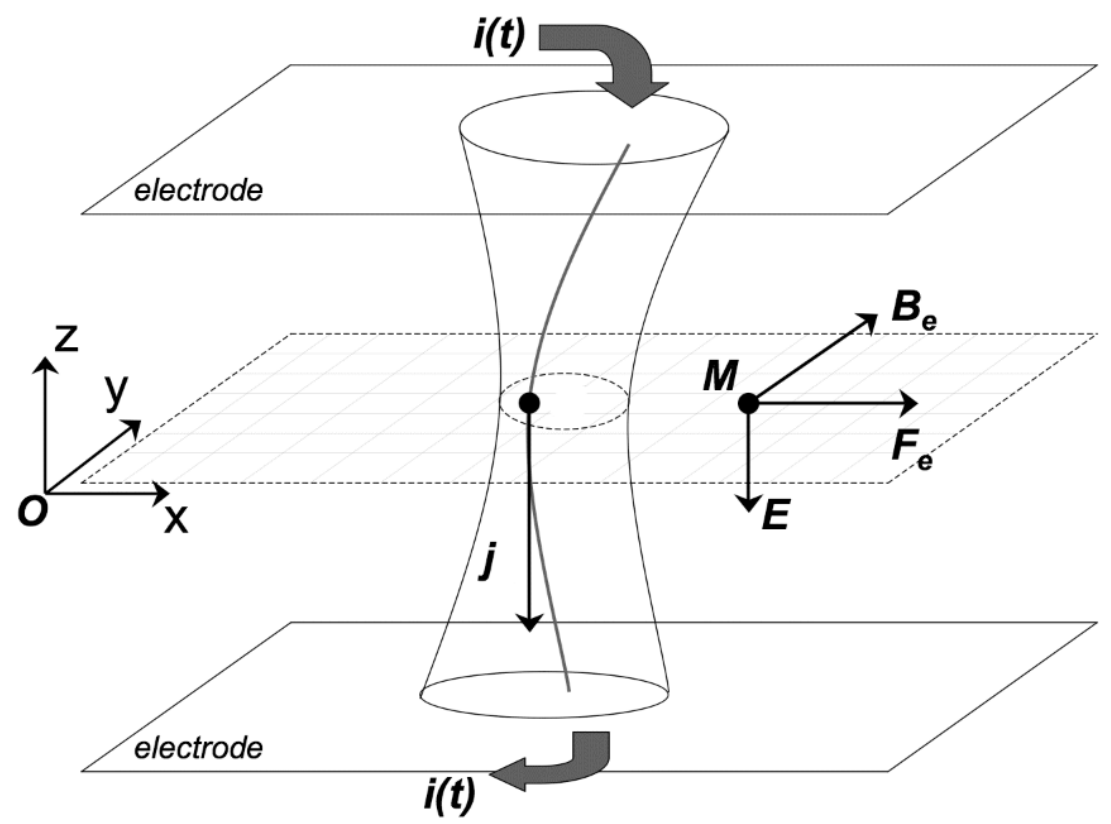

Figure 4: The $(x, y)$ plane used for 2D transient simulations. 


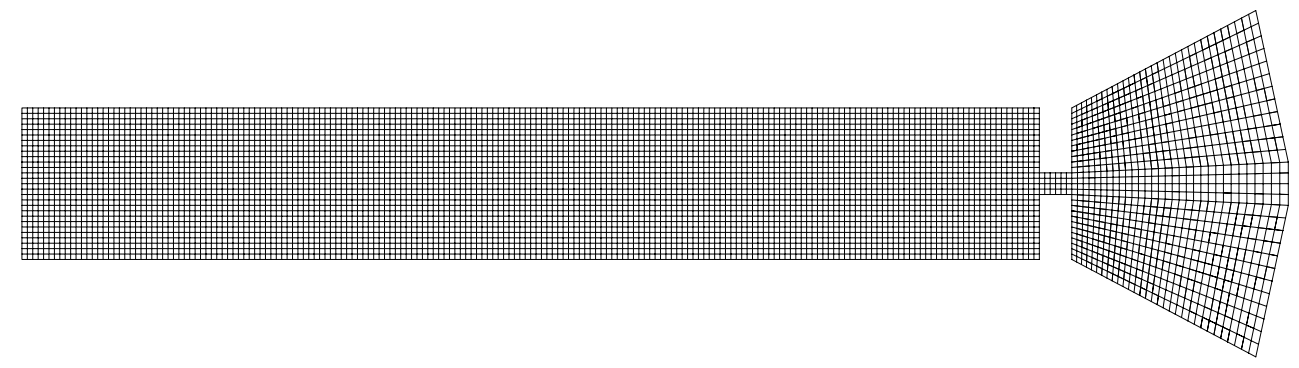

Figure 5: The geometry and grid mesh used for the coupled 2D simulations 

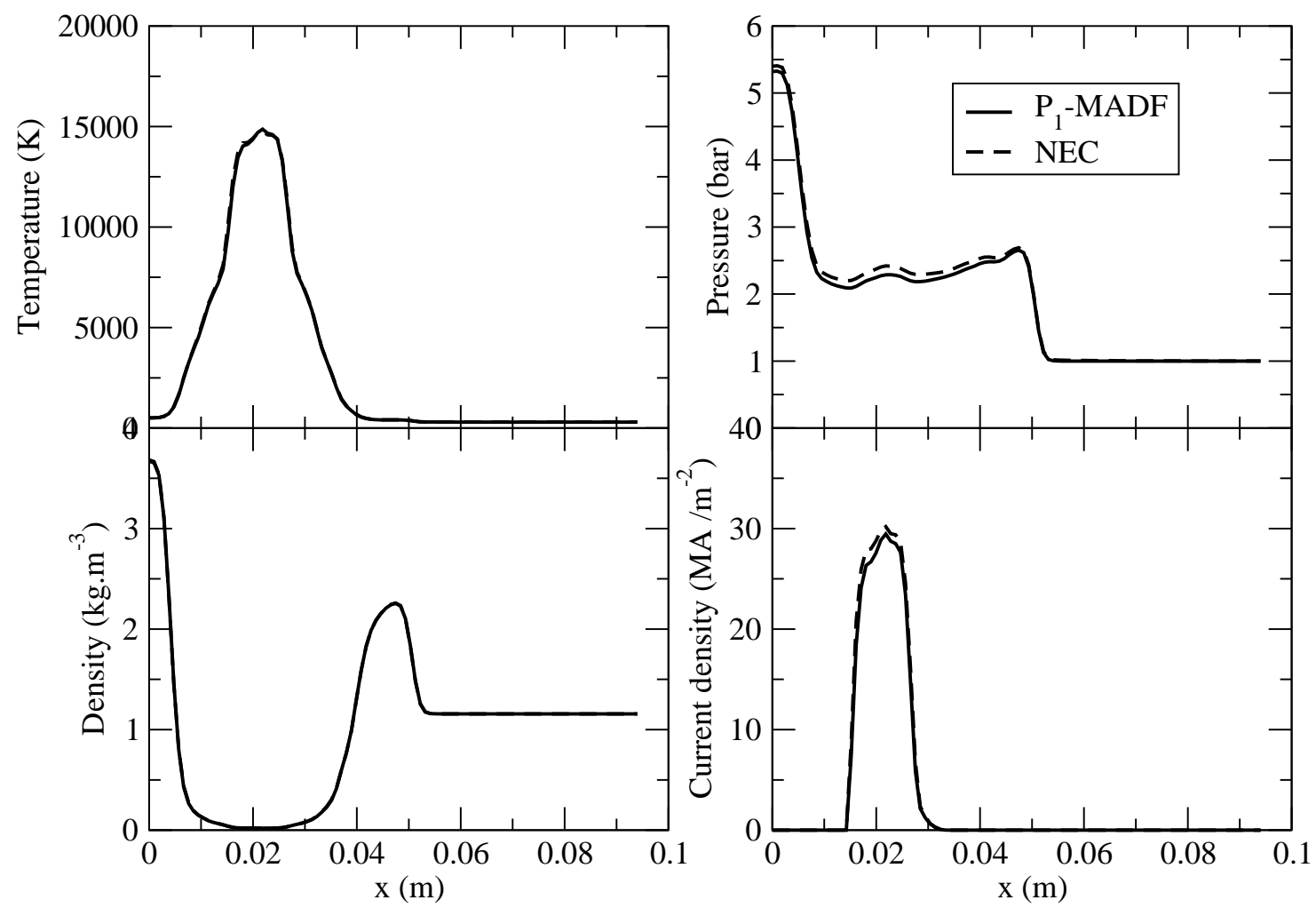

Figure 6: Temperature, pressure, density and current density profiles along the central channel axis calculated with the two radiation models at $t=50 \mu \mathrm{s}$ 

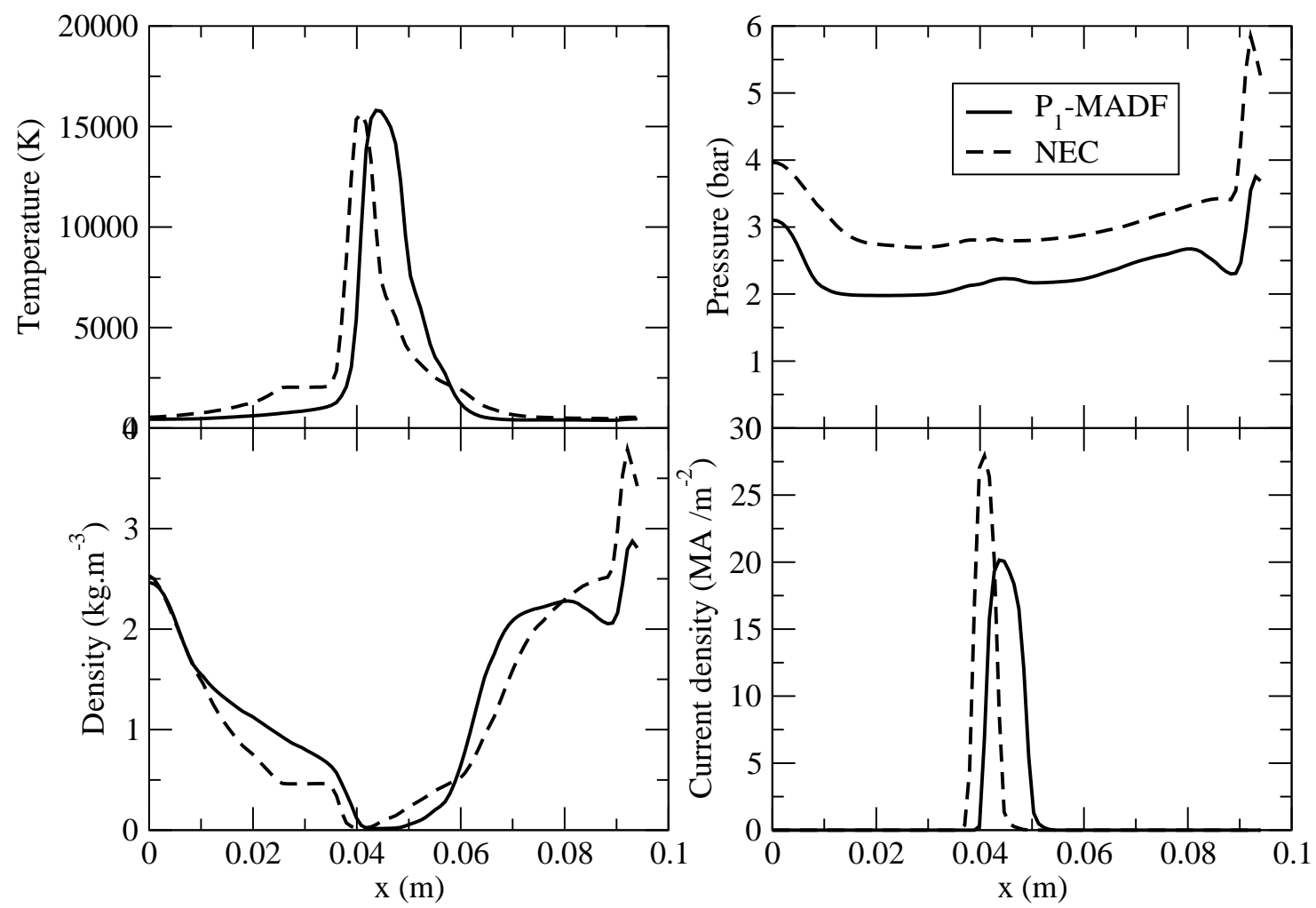

Figure 7: Temperature, pressure, density and current density profiles along the central channel axis calculated with the two radiation models at $t=140 \mu \mathrm{s}$ 

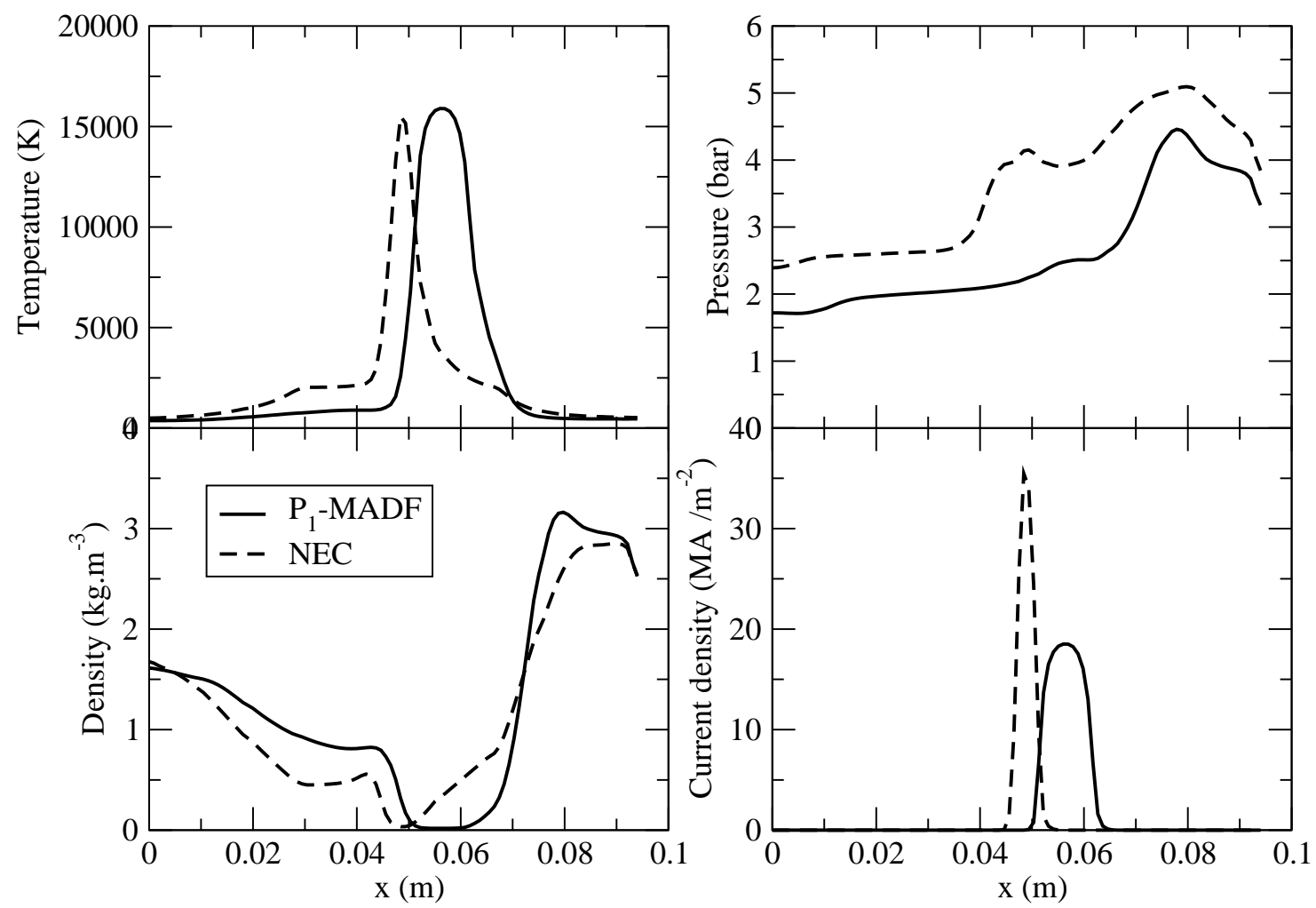

Figure 8: Temperature, pressure, density and current density profiles along the central channel axis calculated with the two radiation models at $t=210 \mu \mathrm{s}$ 

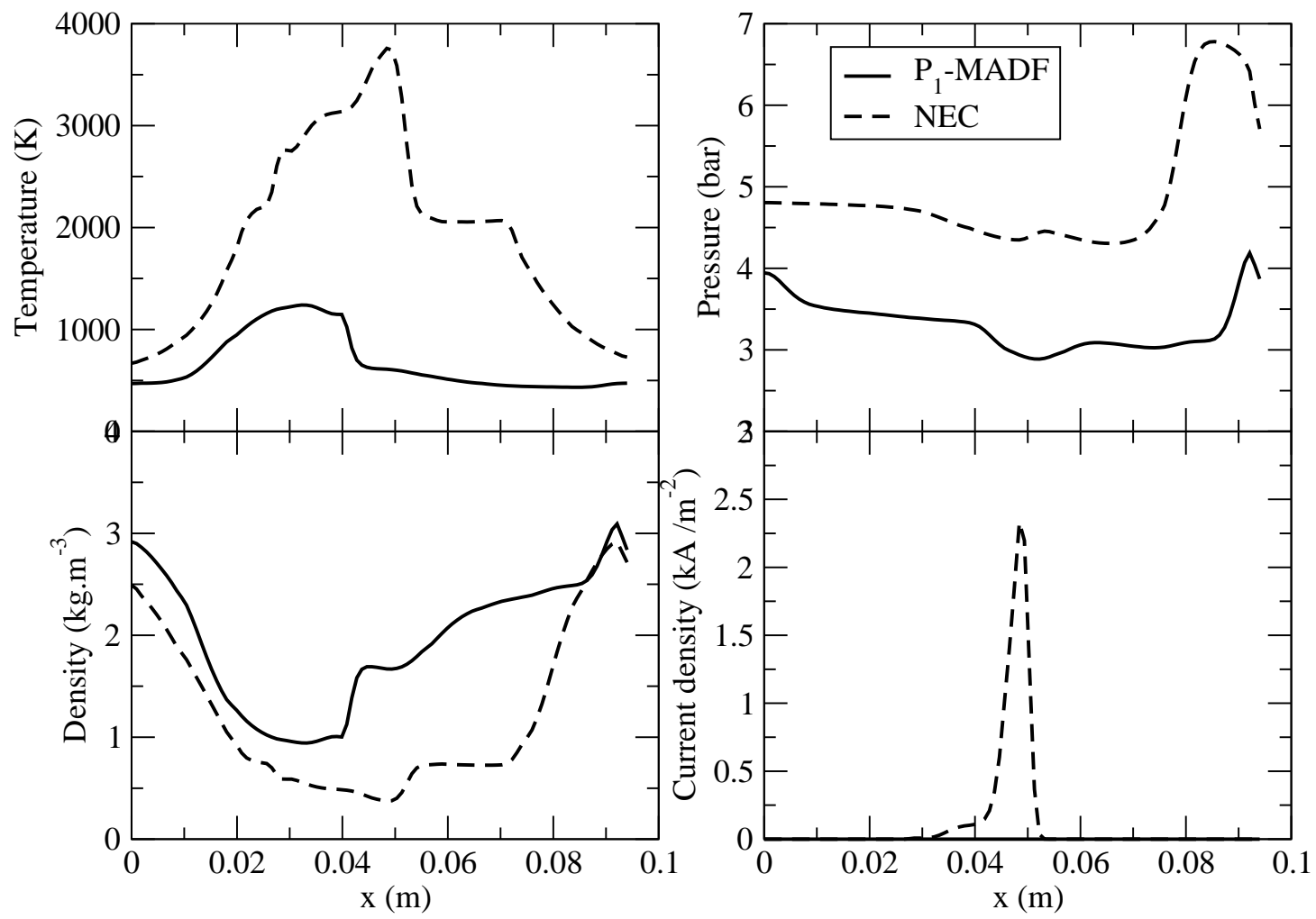

Figure 9: Temperature, pressure, density and current density profiles along the central channel axis calculated with the two radiation models at $t=360 \mu \mathrm{s}$ 


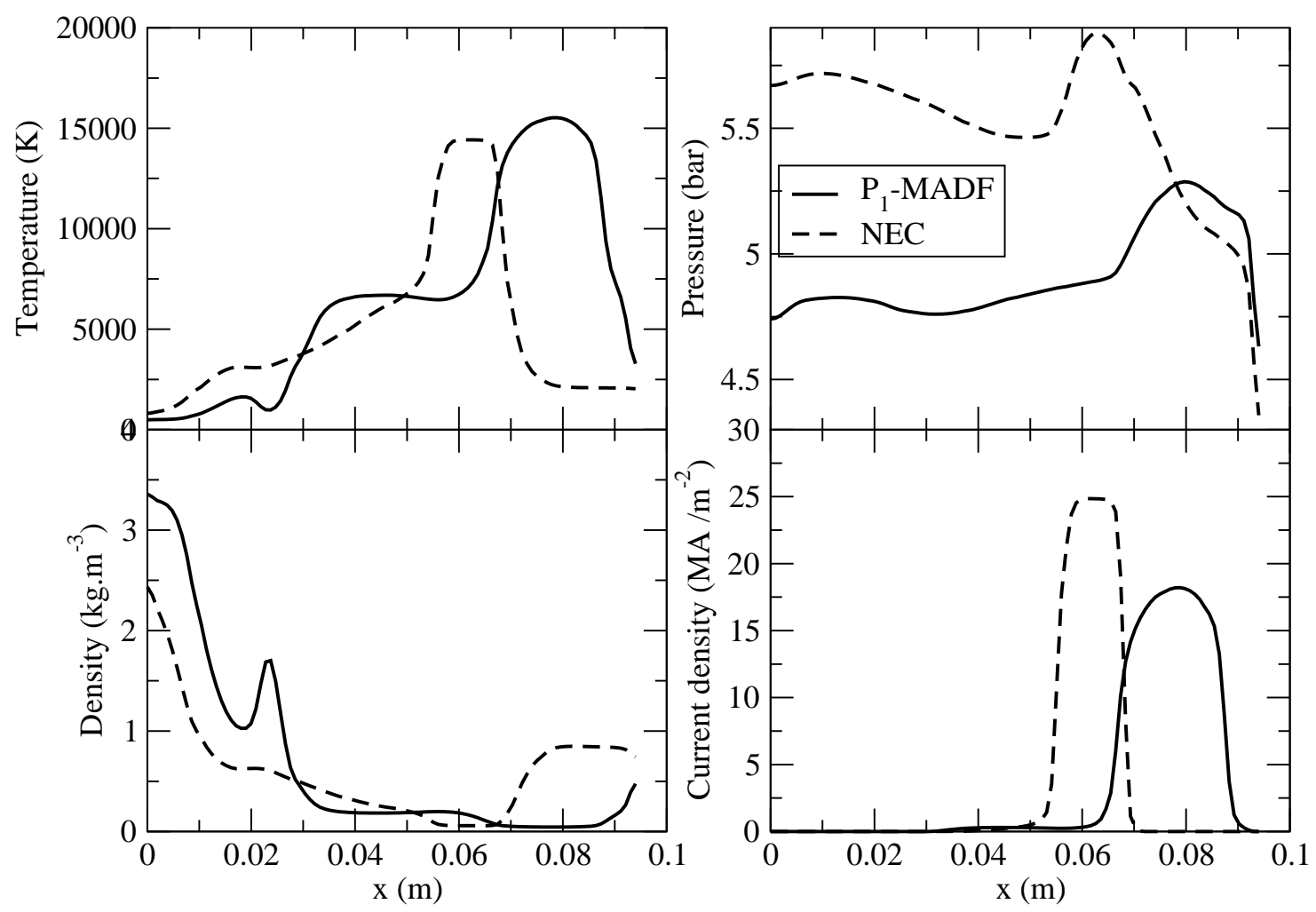

Figure 10: Temperature, pressure, density and current density profiles along the central channel axis calculated with the two radiation models at $t=750 \mu \mathrm{s}$ 


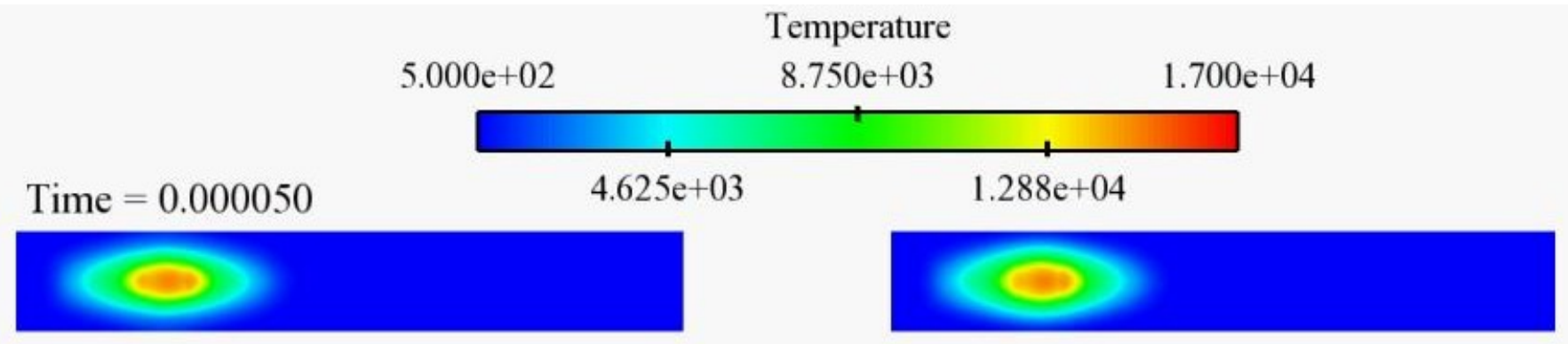

Time $=0.000140$
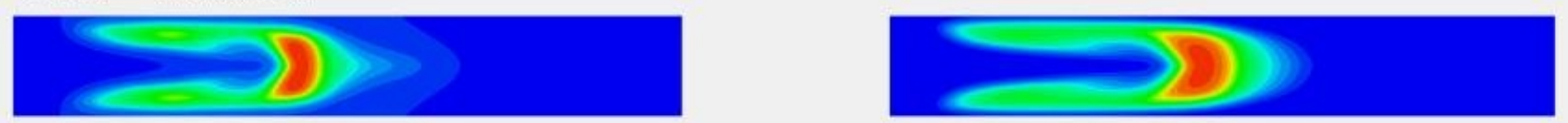

Time $=0.000210$
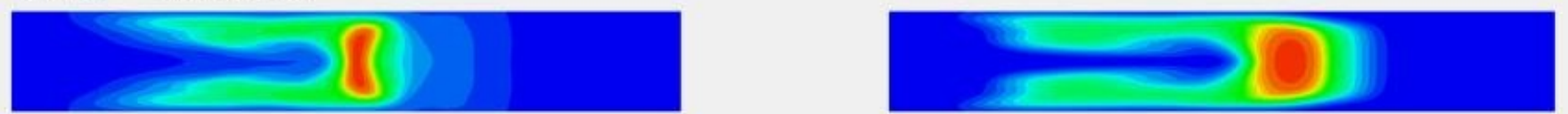

Time $=0.000360$
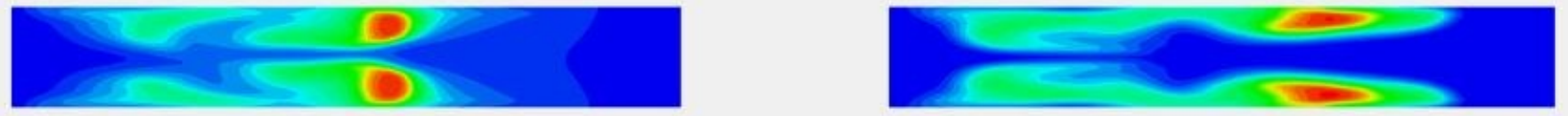

Time $=0.000750$
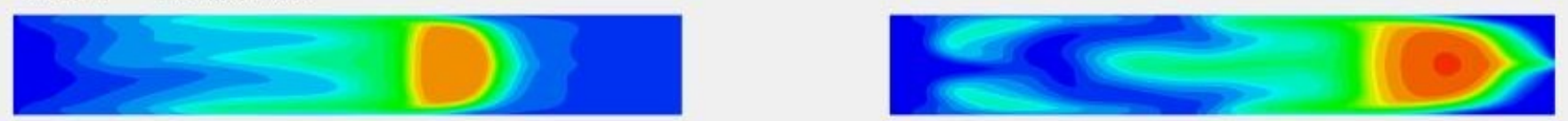

Figure 11: Temperature field computed with NEC (left) and $\mathrm{P}_{1}$-MADF (right) models at different times. 


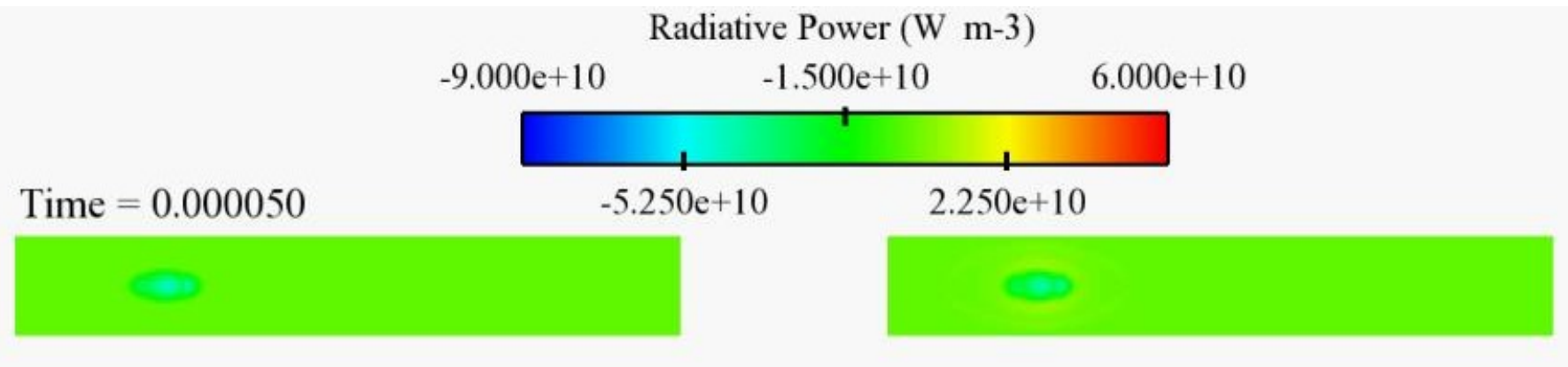

Time $=0.000140$
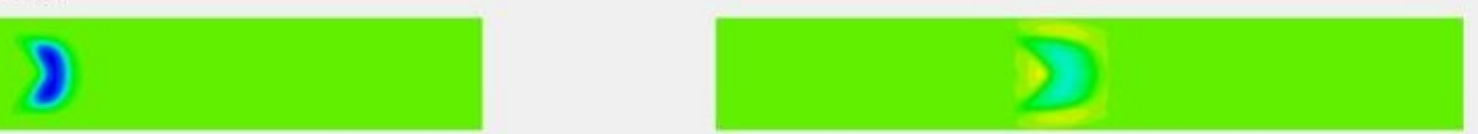

Time $=0.000210$
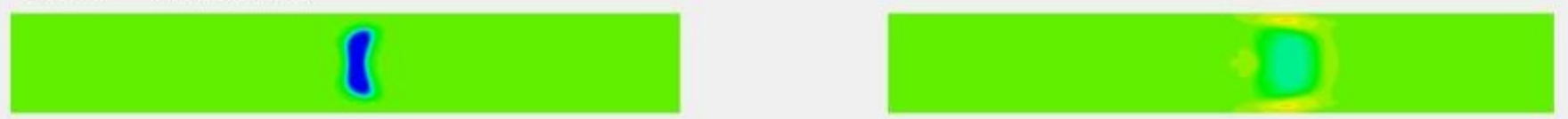

Time $=0.000360$
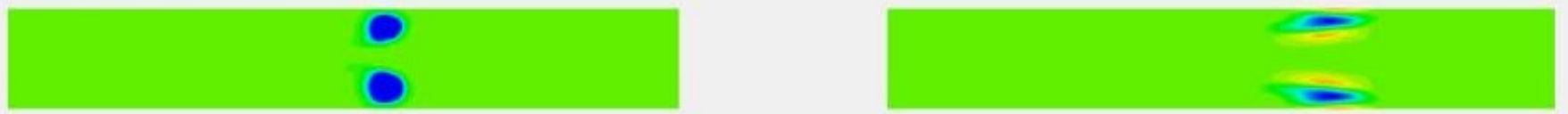

Time $=0.000750$

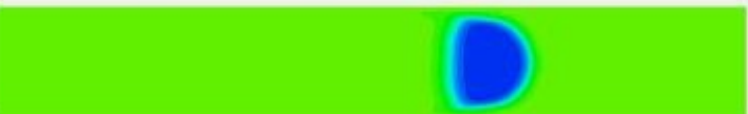

Figure 12: Radiative volumetric power computed with NEC (left) and $\mathrm{P}_{1}$-MADF (right) models at different times. 


\section{List of Tables}

1 Comparison between $\mathrm{P}_{1}$-MADF and NEC CPU times. . . . . . . . . . . 36 


\begin{tabular}{|c|c|c|}
\hline$P_{1}$ method & MADF 36 & MADF 216 \\
\hline radiation calculation each $\Delta t_{\text {flow }}$ & $21239 \mathrm{~s}$ & $90515 \mathrm{~s}$ \\
\hline radiation calculation each $5 \Delta t_{\text {flow }}$ & $4159 \mathrm{~s}$ & $13889 \mathrm{~s}$ \\
\hline radiation calculation each $50 \Delta t_{\text {flow }}$ & $2027 \mathrm{~s}$ & $3959 \mathrm{~s}$ \\
\hline Net emission coefficient & \multicolumn{2}{|c|}{$947 \mathrm{~s}$} \\
\hline
\end{tabular}

Table 1: Comparison between $\mathrm{P}_{1}-\mathrm{MADF}$ and NEC CPU times. 